\title{
Civilised Behaviour: A Chinese Indigenous Intergroup Perception Dimension
}

\author{
Xuan Liu, ${ }^{1, *}$ Wen Shan, ${ }^{2,3, *}$ and Shenghua Jin ${ }^{2}$ \\ 1 School of Information Management, Nanjing University, Nanjing, China \\ 2 School of Psychology, Beijing Normal University, Beijing, China \\ 3 Nanyang Business School, Nanyang Technological University, Singapore
}

\begin{abstract}
$\mathrm{n}$ order to check whether (un)civilised behaviour can be a valid indigenous intergroup comparison dimension for Chinese people, three studies were conducted based on stereotypic explanatory bias (SEB). Study 1 examined the media representation of Chinese and Western (un)civilised behaviours, and the SEB results suggested ingroup derogation of Chinese people regarding civilised behaviour. Study 2 aimed to use a more empirical approach to further analyse Chinese intergroup bias for civilised behaviour at both implicit and explicit levels. Chinese participants' SEB results indicated ingroup derogation and outgroup favouritism were at the implicit level; however, ingroup favouritism and outgroup derogation were at the explicit level. Study 3 was designed to examine how stereotypes that were verified in Study 2 would be changed by manipulating the presentation of extreme, stereotype-relevant information in the form of an internet blog. These findings suggest that (un)civilised behaviour can be a valid indigenous intergroup comparison dimension for Chinese people who perceive themselves as a disadvantaged group compared to Westerners on this dimension. Finally, theoretical contribution and practical implications for social change are discussed.
\end{abstract}

Keywords: civilised behavior, Chinese intergroup bias, stereotypic explanatory bias, intergroup perception, media representation

China is a land of etiquettes and propriety.

Li yi zhi bang, The Commentary of Gongyang, recorded during the Former Han Dynasty, 202 BCE-9 CE.

Representing the origin of Chinese civilisation $(\mathrm{Bu}$, 2007) and the differentiation between human and animal in Chinese ancient thoughts (Book of Rites, Li Ji), propriety (li) is one of the two cores of Confucianism (the other one is ren: morality or benevolence; Wang, 2004). Propriety refers to the established standards of civilised behaviour and, in modern times, propriety in public is known as 'civilised behaviour'.

Civilised behaviours are characterised by conformity to the expectations and requirements for public behaviour according to local propriety, such as behaviours protecting the environment, observing social order, and specifically, offering one's seat to a pregnant woman in a crowded bus. Uncivilised behaviour is the opposite concept, such as behaviours that damage the environment, stripping to the waist in the street, and queue-jumping.
Civilised behaviour is an important indigenous concept in China. The Chinese government attaches great importance to citizens' (un)civilised behaviour and regards it as explicitly associated with the whole nation's international image (Pál, 2009). The Chinese mass media also pays much attention to (un)civilised behaviour, especially when it is concerned with the national image or the impression that Chinese people leave on foreigners (Liu, 2011). Consistently, Chinese citizens are also sensitive to the topic of (un)civilised behaviour, especially when they compare themselves to foreigners.

To some extent, impressions and judgments about social groups are based upon observable group members' behaviour. Kashima (2014) proposed a grounding model of cultural transmission that described how information was deliberately or inadvertently transmitted in everyday joint activities. When this information is further generalised to a large collective and disseminated by repeating it through social networks, the social reality of the collective that we take for granted is maintained. Individuals 
consider themselves as belonging to an ingroup with a certain identity, and this collective identity becomes their social identity (Kashima, 2014). As an important indigenous concept in China, (un)civilised behaviour therefore may have the potential to be a unique dimension of intergroup perception of Chinese people. Despite its attention from the whole of Chinese society and its emphasis in Chinese culture, (un)civilised behaviour remains a neglected topic in psychological research. Our studies, based on Chinese samples, seek to fill in this gap by addressing how Chinese people view themselves on this dimension as compared to Western counterparts.

\section{Chinese Intergroup Bias of Civilised Behaviour}

In previous literature, there are three well-established intergroup comparison dimensions, namely competence, warmth, and morality (Leach, Ellemers, \& Barreto, 2007). Civilised behaviour cannot be covered by any of them. According to the Book of Rites (Li Ji), propriety (li) is an external manifestation of internal morality (ren). It indicates that (un)civilised behaviour is discussed at the level of external and observed behavioural manner, while the judgment of morality satisfies perceivers' wondering about other's inner intentions (Cuddy et al., 2009). However, civilised behaviour not only signals an actor's public morality, but also manifests partial but not all characteristics of warmth, as it includes helping and polite behaviours, but does not include sensitive, expressive, and caring behaviours, which are the specific traits of warmth (Rudman, Greenwald, \& McGhee, 2001). Civilised behaviour also includes actions regarding public rules, such as waiting for the traffic light to turn green before crossing a street.

The unique characters manifested by civilised behaviour make it a judgmental cue in China, which is a Confucian country. In Confucian ideologies, different social and economic hierarchies have had different levels of propriety (The Book of Hsun Tzu, Historical Records). In ancient times in China, propriety served to differentiate citizens' social and economic classes ( $\mathrm{Du}, 2006)$. For instance, the noblemen with a higher social and economic status were deemed to behave with more propriety than ordinary people. In modern society in China, the advantaged group with higher power and status is still assumed to be more civilised than the disadvantaged groups (Tomba, 2009).

With clear differences between advantaged and disadvantaged group members' intergroup bias pattern, the only question that remains is how Chinese people perceive their ingroup status compared to an outgroup. Research has demonstrated that people tend to use history to understand why the world is the way it is (e.g., Liu \& Atsumi, 2008). According to this argument, Chinese people's national intergroup attitudes might be influenced by the representation of long-term economic poverty and a disadvantaged status in modern history. Throughout mod- ern history, the West was not only assumed to be militarily 'superior' in the 19th century, but increasingly came to be perceived as being advantaged in administration ( $\mathrm{Liu}, \mathrm{Li}$, \& Yue, 2010; Pye, 1996). By contrast, in the treaty-port concessions, all members of Chinese society - Manchu or Han, nobility or commoners - were considered as inferior by the West (Liu et al., 2010).

Chinese people might identify themselves as a disadvantaged group and perceive the Western outgroup as an advantaged group and with higher social status. Previous literature has provided certain support for this argument. Chinese people in Malaysia have developed outgroup-favouring and ingroup-derogating attributions for warmth and morality (Hewstone \& Ward, 1985), while Chinese people in the United States have exhibited implicit ingroup derogation on morality (Ma-Kellams, SpencerRodgers, \& Peng, 2011). To test whether civilised behaviour also has the potential to be Chinese people's intergroup perception dimension by showing the same pattern found in previous literature, three studies with different approaches were conducted.

The grounding model (Kashima, 2014) provides an explanation of how cultural constructs such as stereotypes and social perception are developed by individuals in society (Liu, 2014). The grounding model indicates that given cultural information might be questionable if it is inaccurate. Inadvertently, essentialist beliefs may satisfy the need for cognitive security and the need to belong to a group (Chao \& Kung, 2014, 2015). However, essentialist beliefs about the inherent differences between social groups (e.g., racial group, cultural group) might be oversimplified and misunderstood. Cognitive rigidity, prejudices, biased stereotypes, and intergroup biases by the general public might be the byproduct of essentialist beliefs (Chao \& Kung, 2014, 2015).

Biased stereotypes of Chinese people (e.g., ingroup derogation and outgroup favouritism) might produce many negative social psychological phenomena, such as lower self-esteem in the presence of an outgroup, and being afraid of being assimilated by an outgroup culture. Reducing biased stereotypes and effecting interventions for social change that benefit society and individuals are meaningful and imperative. Proper guidance from the mass media might help change biased stereotypes of Chinese people. Further, adding more content about Chinese traditional culture, such as Confucian philosophy, when reporting (un)civilised behaviours might help improve civilised behaviour and inhibit uncivilised behaviour of Chinese people.

\section{Overview of the Present Study}

In Study 1, content analysis of internet news reports on (un)civilised behaviour about Chinese intergroup perception was examined. Specifically, we focused on how Chinese and Western (un)civilised behaviours were overrepresented or underrepresented, and the occurrence of internal and external attributions of Chinese 
(un)civilised behaviour. Study 2 sought to examine whether (un)civilised behaviour is indeed a qualified dimension of intergroup perception for Chinese people by using an implicit SEB measure and explicit self-report scale. Study 3 aimed to change Chinese group bias (verified in study 2) by manipulating the presentation of extreme stereotype-relevant information in the form of internet blogs.

\section{Study 1}

Stereotype and evaluative intergroup bias does not develop in isolation, but as a complex function of various influences in a broader sociocultural context. Our memories and perceptions in intergroup comparisons are often associated with the way they are reported in the media, which forms our taken-for-granted frameworks of understanding (Frewin, Pond, \& Tuffin, 2009).

\section{Mass Media's Bias}

Media representations often offer a particular and usually dominant way of understanding the world with biased reports. These reports characterise stereotyped groups and provide prescriptions for addressing social concerns on stereotype-consistent events. For example, a blog criticised tourists riding the charging bull in Wall Street (Wang, 2007), but neglected mentioning Westerners who did the same thing. This ignited a heated debate on the internet about Chinese uncivilised behaviour. For citizens and the government, Chinese uncivilised behaviour is viewed as causing the whole country to suffer from shame and humiliation (Chinese Foreign Ministry, 2007). It was also pointed out that the biased news reports and the attitudes of the followers are derived from 'a deep self-abasing and a strong worship to Westerners' (Wan, 2009).

\section{Social Identity Theory}

Social identity theory (SIT) argues that people have an inbuilt tendency to categorise themselves into ingroups, building a part of their identity on the basis of membership of that group and enforcing boundaries with other groups (Tajfel \& Turner, 1986). Meanwhile, people strive to maintain a positive image of their group in order to maximise positive distinctiveness and achieve a positive self-esteem (Tajfel \& Turner, 1986). However, SIT was only tested to be valid among perceived 'superior' groups, or at least among groups with equal status compared to a given group (Lam, Ahearne, Hu, \& Schillewaert, 2010). For disadvantaged groups, ingroup favouritism and outgroup derogation were only exhibited by explicit measures (Jost, Pelham, \& Carvallo, 2002). At an implicit level, however, an impressive body of literature has demonstrated that disadvantaged groups favoured an ingroup and derogated perceived 'superior' outgroups (e.g., Haye et al., 2010), for which SIT failed to adequately account.

\section{Stereotypic Explanatory Bias}

For content analysis in Study 1 and implicit intergroup bias measure in Study 2 and Study 3, stereotypic explanatory bias (SEB; Von Hippel, Sekaquaptewa, \& Vargas, 1997) was used for our methodology and assumptions. In much of the psychological literature, SEB has been applied as an implicit measure, which reflects the unintended influence of stereotypes on processing (e.g., Logel et al., 2009). SEB has also been acknowledged by researchers as a valid, implicit stereotyping measure, with a high ecological validity and a good predictive capability of behaviours (e.g., Gonsalkorale, von Hippel, Sherman, \& Klauer, 2009). The foundation of SEB includes two validated theories (e.g., Evett, Devine, Hirt, \& Price, 1994). The first one indicates that people are more likely to make attributions for behaviours that are contradictory to their expectations in order to make sense of the behaviours (Hastie, 1984). The second theory states that people tend to provide situational attributions rather than dispositional attributions when their expectation of one's behaviour is different from the actual behaviour (Kulik, 1983). Consequently, SEB indicates the tendency to spontaneously offer more attributions for stereotypeinconsistent behaviours than for stereotype-consistent behaviours, and also the tendency to provide more situational explanations for stereotype-inconsistent behaviours and more dispositional explanations for stereotypeconsistent behaviours. Thus, if participants perceive that Chinese people behave in an uncivilised way, namely ingroup derogation of civilised behaviour, they will provide more (especially external) attributions for Chinese civilised behaviours and fewer attributions for their uncivilised behaviours. If participants hold the stereotype that Westerners behave in a civilised way, namely outgroup favoritism for civilised behaviour, they will provide more (especially external) attributions for Western uncivilised behaviours and fewer for their civilised behaviours.

Despite the meaningful theoretical implications, little previous research, if any, has identified civilised behaviour as an indigenous intergroup perception dimension in China. Our study is the first to fill this gap by conducting a media content analysis. Consistent with the theories in the literature review, the following hypotheses were proposed:

Hypothesis 1a: Compared to Westerners, Chinese civilised behaviours are underrepresented and Chinese uncivilised behaviours are overrepresented.

As a methodological innovation, in Study 1 , the content analysis was conducted using the theoretical hypotheses of SEB. According to SEB, the following hypotheses were developed:

Hypothesis 1b: Attributions for Chinese civilised behaviours are overrepresented compared to attributions for Chinese uncivilised behaviours. 
Hypothesis 1c: For Chinese civilised behaviours, internal attributions are underrepresented and external attributions are overrepresented, whereas for Chinese uncivilised behaviours, internal attributions are overrepresented and external attributions are underrepresented.

\section{Materials and Methods}

The sampled materials refers to all the news stories covering Chinese and Western civilised and uncivilised behaviours from China's top two highly visited news websites (news.sohu.com and news.qq.com) and was generated by a keyword search without limits on the published date. The five keywords used for selection of articles were civilised behaviour (wenmingxingwei), behaviour quality (suzhi), Chinese people (zhongguoren), Westerner (xifangren), and Occidental (yangren). For each search, we combined one of the first two words and one of the last three words. After deleting those articles unrelated to Chinese or Western (un)civilised behaviour, the valid sohu sample contained 109 news stories, and the qq sample contained 82 news stories, published from November 2003 to March 2009.

Coding scheme. For each news article, two independent coders who were familiar with the present subject examined the codes. The code of the whole theme refers to the answer to: 'What does the article talk about?' When coders judged that an article mainly mentioned Chinese civilised (or uncivilised) behaviour, the theme code was 'Chinese: civilised' or 'Chinese: uncivilised', following the same rule as 'Western: civilised' and 'Western: uncivilised'.

The attribution code refers to the answer to: 'What reasons does the article provide for why the actors behaved like that?' Coders perused every article and searched for the reasons why Chinese and Westerners behaved in an (un)civilised way. The numbers of external and internal attributions were counted respectively for each kind of behaviour. Here, internal attribution is defined as an explanation of (un)civilised behaviour based upon inferences about the individual's inherent personality, attitude, disposition, ability, or effort (Heider, 1958). For example, 'The man jumped the queue because he is rude and does not consider others' was categorised as the internal attribution. External attribution refers to an explanation of (un)civilised behaviour based upon inferences about the external source, such as other people and various environmental stimuli (Heider, 1958). For example, 'The man did not wait for the green light to cross the street due to the problem of the traffic light' was categorised as an external attribution.

Intercoder reliability. A detailed coding manual was discussed and agreed upon by the two coders and the present authors. Due to simplicity, the theme code was completely congruent. Intercoder agreement in attribution code was over $90 \%$. Disagreements between coders were resolved by an open group discussion with the authors.

\section{Results and Discussion}

\section{Representation of In- and Outgroup Civilised and Uncivilised} Behaviour

For Chinese people, 6 articles mentioned civilised behaviours and 159 articles mentioned uncivilised behaviours; for Westerners, 11 articles mentioned civilised behaviours and 11 mentioned uncivilised ones, $\chi^{2}(1, n=$ $187)=50.49, p<.001$. Consistent with our assumptions, compared to the Westerners, Chinese uncivilised behaviours were overrepresented while Chinese civilised behaviours were underrepresented. This provides evidence that Chinese journalists hold negative stereotypes about Chinese behaviours.

The equal occurrence frequency appears to suggest that a non-biased representation of the attitudes content implicates outgroup favouritism. Unlike the articles that mentioned Chinese uncivilised behaviours, which aimed to criticise Chinese public manners and proposed how these could be improved, most of the articles mentioning Western uncivilised behaviours aimed to disprove the widely accepted notion that only Chinese people are uncivilised and that Chinese should learn from Westerners about how to be civilised. Furthermore, unlike the articles reporting on Chinese civilised behaviours that attempted to demonstrate the effectiveness of the measures for improving Chinese civilisation, most of the news reports that mentioned Western civilised behaviours were encouraging Chinese people to learn from Westerners and to be ashamed of their own uncivilised behaviours.

\section{Representation of Attributions for Chinese Civilised and Uncivilised Behaviour}

As Hypothesis $1 \mathrm{~b}$ predicted, attributions were overrepresented for Chinese civilised behaviours and underrepresented for Chinese uncivilised behaviours. For 159 Chinese uncivilised behaviours, 92 attributions were provided (0.58 per Chinese uncivilised behaviour), whereas for 6 Chinese civilised behaviours, 12 attributions were provided ( 2 per Chinese civilised behaviour).

In addition, compared to external attributions, internal attributions were overrepresented for Chinese uncivilised behaviour and underrepresented for Chinese civilised behaviour. For Chinese civilised behaviour, 25\% $(n=3)$ of attributions were internal and $75 \%(n=9)$ were external; for Chinese uncivilised behaviour, $67 \%(n=$ 59) of attributions were internal and $33 \%(n=29)$ were external, $\chi^{2}(3, n=100)=76.48, p<.001$. This result supported Hypothesis $1 \mathrm{c}$. According to SEB, these results suggest that the stereotype that Chinese people are uncivilised is represented in Chinese mainstream internet media.

The amount of attributions to Western behaviours was not involved in the analysis because of a lack of an adequate sample size (total $N=3$ ).

In Study 1, content analysis of the internet news reports on (un)civilised behaviour about Chinese 
Table 1

Distribution of Internal and External Attributions for Chinese Civilised and Uncivilised Behaviours

\begin{tabular}{lll}
\hline & $\begin{array}{l}\text { Civilised behaviours } \\
\text { (percentile) }\end{array}$ & $\begin{array}{l}\text { Uncivilised behaviours } \\
\text { (percentile) }\end{array}$ \\
\hline Internal attribution & $3(25 \%)$ & $80(91 \%)$ \\
External attribution & $9(75 \%)$ & $8(9 \%)$ \\
\hline
\end{tabular}

intergroup perception was conducted. Results indicated that Chinese journalists hold negative stereotypes about Chinese civilised behaviours and the stereotype that Chinese people were uncivilised was represented in Chinese mainstream internet media. Meanwhile, attributions were overrepresented for Chinese civilised behaviours and underrepresented for Chinese uncivilised behaviours.

\section{Study 2}

Study 1 demonstrated that Chinese internet media viewed their ingroup as uncivilised and perceived the Western outgroup as more civilised than the ingroup. Study 2 aimed to use a more empirical approach to examine Chinese intergroup bias of civilised behaviour and check the detail of the intergroup bias at both an implicit and explicit level. An implicit SEB measure and an explicit self-report scale were developed based on those typical civilised or uncivilised behaviours from internet news reports that were analysed in Study 1. If civilised behaviour is a qualified dimension of intergroup perception, Chinese participants should exhibit ingroup derogation and outgroup favouritism in the implicit measure, as well as ingroup favouritism and outgroup derogation in the explicit measure.

According to SEB, the following hypotheses were proposed:

Hypothesis 2a: In an implicit SEB measure, Chinese participants conceive more attributions for Chinese civilised behaviours than for Chinese uncivilised behaviours, but conceive fewer attributions for Western civilised behaviours than for Western uncivilised behaviours.

Hypothesis 2b: In an implicit SEB measure, Chinese participants conceive more external attributions and fewer internal attributions for Chinese civilised behaviours than for Western civilised behaviours.

Besides the implicit SEB measure, a self-report scale was also included to measure participants' explicit intergroup perception of civilised behaviour. Previous research suggests that social recognitions and intergroup processes could be influenced by a self-protection motive, such as discrimination between the ingroup and outgroup and the emotions for outgroup (Maner, Miller, Moss, Leo, \& Plant, 2012). According to SIT and self-protection theory, the following hypothesis was developed:

Hypothesis 2c: Chinese participants perceive ingroup members as behaving in a more civilised manner than Western outgroup members in the explicit self-report scale.

\section{Materials and Methods}

Participants. Chinese internet users $(N=109)$ who volunteered to be participants for free were recruited via the internet. After eliminating 13 invalid responses (i.e., those that were finished in less than 3 minutes, or were filled with irrelevant words, punctuation, or copies of the item sentences), 96 responses (51 males, 45 females, mean age 25.32 years) were considered valid.

The implicit SEB measure for intergroup bias. A pretest was conducted to select civilised and uncivilised behaviours for the SEB measure. As an alternative for primary SEB items, 16 typical civilised behaviours and 16 typical uncivilised behaviours without overlap were selected from the internet news reports used in Study 1.

An independent sample of 14 graduates majoring in psychology (4 men and 10 women) rated each of 41 behaviours (16 civilised, 16 uncivilised, and 9 neutral) on how civilised they were on a 7-point scale $(1=$ highly uncivilised, $7=$ highly civilised). Behaviours that met the following statistical criteria were selected for the formal SEB measure. First, a behaviour used to represent civilised behaviours must have been rated as significantly more civilised than the average rating of the neutral behaviours and vice versa for an uncivilised behaviour. Second, the extent of civilisation of the behaviours described in SEB items must be counterbalanced across different groups. Specifically, each behaviour was labelled with either a Chinese or Western name, and the statistical difference of the ratings between groups should be insignificant. Eight civilised and eight uncivilised behaviours were selected for the formal SEB measure.

The formal SEB measure presented a set of 25 items. Each item described a target person engaging in a behaviour. Eight items described behaviours that were consistent with the hypothetic stereotypes (i.e., ingroup derogation and outgroup favoritism; e.g., 'Zhao Qi is carving his name on a public sculpture with a key, because ...'; 'Henry is waiting for the traffic light, although there's no car on the street, because ...'), and 8 items for stereotype-inconsistent (i.e., outgroup derogation and ingroup favouritism; e.g., 'Tom is throwing a piece of paper out of the car window, because ...'; ' $\mathrm{Li}$ Hao is opening a door for a lady, although he could go through it ahead of her, because ...'). The remaining nine items were neutral with respect to (un)civilised behaviours (e.g., 'James is cooking the dinner'). To protect the implicit characteristic of SEB, instead of explicitly labelling group membership, a Chinese name suggested an ingroup member and an English name suggested an outgroup member.

An 11-point Likert scale of evaluation of the civilisation degree of the actors was used to validate the extremity of the experimental material, similar to a scale used by Kunda and Oleson (1997).

The explicit self-report scale for intergroup bias. A 16item self-report, rated on a 6-point Likert-like scale, was developed to measure the intergroup bias based on the 
explicit comparison of (un)civilised behaviours. The dimensions of the scale included civility, public morality, observance of social norms/order, and modest manners. Sample items were: 'Most foreigners are ethical', 'In China, most people do not adhere to social order', 'In the West, the level of development of social public morality is higher than in China'. This comparative construction of the scale determined the testing method that a score significantly less than 3.5 (the middle of 1-6 points) reflected that the participant believed Chinese were more civilised than Westerners. The internal consistency reliability (alpha coefficient) was 0.898 and the self-assessment grading criterion-related validity (with the grading criterion item, 'The Westerners are more civilised than Chinese') was $0.672(p<.001)$

Procedure. The experiment was administered via the internet. After logging into the testing web page, a set of standardised instructions emphasising non-normal answers was presented in Chinese. Then, the SEB items appeared in a random order. Participants could see the following sentence item only if they completed the former one. When all SEB items were finished, participants were invited to fill in the self-report scale. Upon completion, participants were thanked for their participation.

Calculation of SEB score. The SEB score was calculated by subtracting the number of explanations provided for stereotype-consistent behaviours (i.e., Chinese actors behave in an uncivilised way or Western actors behave in a civilised way) from the number of explanations provided for stereotype-inconsistent behaviours (i.e., Chinese actors behave in a civilised way or Western actors behave in an uncivilised way). Ingroup derogation is thus indicated by providing more attributions for Chinese civilised behaviours than uncivilised behaviours, namely the SEB1. Similarly, outgroup favouritism was indicated by providing more attributions for Western uncivilised behaviours than civilised behaviours, namely the SEB2. As a secondary dependent variable, the SEB3 score refers to the average score computed by subtracting the number of internal attributions from the number of external attributions. Providing more external attributions than internal ones for Chinese civilised behaviours indicated ingroup derogation, and providing more external attributions than internal ones for Western uncivilised behaviours indicated outgroup favouritism.

\section{Results and Discussion}

Chinese intergroup bias in the implicit SEB measure. Responses on the SEB measure were scored by two independent coders who classified each sentence completion as either an explanation or not. The responses judged to be explanations were further scored as internal (i.e., dispositional) or external (i.e., situational) attributions. The judgments of the two coders were highly correlated ( $r s>$ .933 , ps <.001).

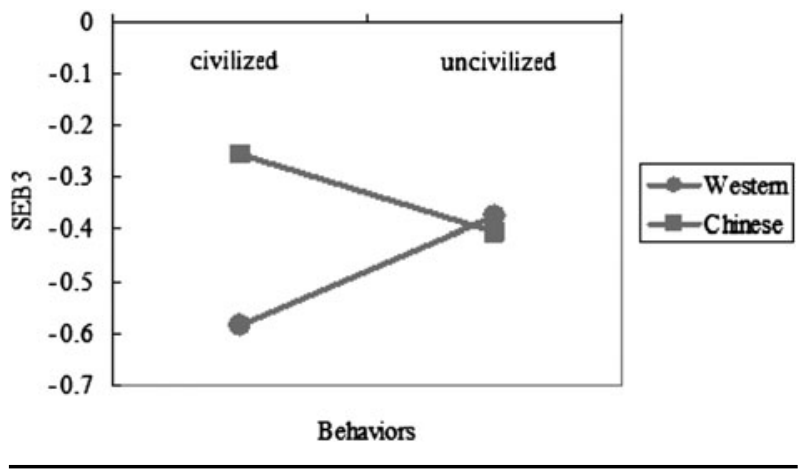

\section{Figure 1}

Chinese ingroup derogation and outgroup favoritism on civilised behaviors. SEB3 (external attribution - internal attribution) as a Function of Whether the Described Behaviors were Civilised or Uncivilised and Whether the Actor in the Sentence was a Chinese or a Westerner. Western actors; - , Chinese actors.

The SEB1 (ingroup derogation) score ranged from -1.75 to $3.00, M=0.260, S D=0.527$, and was significantly greater than zero, $t(95)=4.843, p<.001$. The SEB2 (outgroup favouritism) score ranged from -1.25 to $2.50, M=0.242, S D=0.622$, and was significantly greater than zero, $t(95)=3.812, p<.001$. As Hypothesis 2a predicted for the implicit measure, Chinese participants chose more attributions for Chinese civilised behaviours than for Chinese uncivilised behaviours, but fewer attributions for Western civilised behaviours than for Western uncivilised behaviours. According to the SEB, these results strongly indicate ingroup derogation and outgroup favouritism by Chinese people.

The score of the SEB3 (external attribution-internal attribution) further validated our prediction by supporting Hypothesis $2 \mathrm{~b}$. An ANOVA indicated a significant interaction emerging between whether the depicted behaviours were civilised or uncivilised and whether the actor of the behaviour was Chinese or Western, $F(1,95)=$ 8.591, $p<.005, \eta^{2}=0.08$. Specifically, participants chose more external attributions and fewer internal attributions for Chinese civilised behaviours (SEB3: $M=-.255, S D$ $=1.025$ ) than for Western civilised behaviours (SEB3: $M$ $=-0.583, S D=0.828), F(1,95)=15.61, p<.001, \eta^{2}$ $=0.14$ (see Figure 1). When the depicted behaviour was uncivilised, however, the difference between the ingroup and outgroup was not significant, $F<1$.

According to the SEB, these results suggested a negative stereotype of Chinese civilised behaviours and a positive stereotype of Western civilised behaviours.

Chinese intergroup bias in the explicit self-report measure. Response choices were summed such that higher scores indicated higher ingroup bias. Scores ranged from 1.00 (strongly disagree) to 6.00 (strongly agree), $M=3.204$, $S D=1.048$, and was significantly less than the theoretical average value of 3.5 (Chinese were more civilised than Western), $t(95)=-2.766, p<.008$. Supporting Hypothesis $2 c$, this result indicates that Chinese participants 
explicitly regarded ingroup members as more civilised than the Western outgroup.

\section{Study 3}

The results of Study 2 verified that Chinese people exhibit ingroup derogation and outgroup favouritism for the implicit measure, as well as ingroup favouritism and outgroup derogation for the explicit measure. In Study 3 , we aimed to examine how the stereotypes at both implicit and explicit levels that were verified in Study 2 would be changed by manipulating the presentation of extreme stereotype-relevant information in the form of internet blogs.

Some group stereotypes are established on the basis of incorrect notions and often reflect biased judgments. Taking into account the group as an integral entity, those who perceive group stereotypes often overlook the individual differences among group members, which can result in prejudice. A typical approach to changing stereotypes about a social group is to expose information that disconfirms the stereotype (Wilder, 1993).

\section{The Media's Impact on Stereotypes}

At present, few native Chinese have direct contact with foreigners. As media dependency theorists have stated (BallRokeach \& DeFleur, 1976), the importance of mass media as a knowledge source used in reality construction increases when other sources of knowledge are unavailable. Thus, information via the mass media might be a breakthrough in contributing to studies on stereotype change, as the media are good at manifesting stereotypes. There are a several studies that provide evidence about stereotypes in media portrayal that significantly influence viewers and subsequently judgments of target groups (e.g., Dixon, 2006).

People are inclined to express opinions that agree with the consensus. Actions that are adaptive to the social environment can result in more positive or less negative outcomes (Kashima, 2014). A previous study demonstrated how Australian participants' beliefs about Americans and Australians were strongly influenced by opinions expressed by fellow Australian (Haslam et al., 1996). Similarly, providing feedback to European Americans that others held different beliefs about African Americans significantly changed their pre-existing beliefs (Stangor, Sechrist, \& Jost, 2001). These studies suggest that learning about public opinions has the potential to either produce or inhibit stereotype change.

\section{Subtyping}

Journalists frequently use examples as a powerful tool to add credibility and authenticity to their stories and thus influence news media consumers' perceptions of reality (Gibson \& Zillman, 1993). However, counterstereotypic information is not always powerful enough to influence stereotypes when subtyping occurs. When the examples disconfirm a stereotype in an extreme way, or when counter-stereotypic behaviours performed by group members are thought to be subtypical of the group, these members can become isolated from the group, and then the group's stereotype is maintained (e.g., Weber \& Crocker, 1983). These extreme examples are seen as not credible, and these behaviours are attributed to unstable, situational causes (Wilder, Simon, \& Faith, 1996). In this case, perceptions are not generalised from the deviant examples of the stereotype.

As a rule, people usually attempt to maintain their perceptions of a stereotype by subtyping the stereotypeinconsistent actions of deviant members when the counter-stereotypic examples are extreme and atypical (e.g., Weber \& Crocker, 1983). But when the prestereotype is negative about an ingroup and positive about an outgroup that is not preferred, the 'rule' may be different. The extreme deviant (e.g., a highly civilised Chinese) appears to be not deviant but rather is seen as an exemplar that can prove that not all Chinese are uncivilised. The 'highly uncivilised foreigner' would be regarded as a pleasant finding. So, we argue that the change rule in response to stereotype-inconsistent information may depend on the nature and content of the pre-existing stereotype.

\section{Functions of Emotion and Self-Worth on Stereotype Change}

Stereotyping is a dynamic process, thus the effect of emotion and motive can never be neglected. Many researchers (e.g., Munro \& Ditto, 1997) have been concerned with the importance of emotion in the formation and maintenance of negative intergroup attitudes.

Exposure to counter-stereotypic information might produce a negative affective reaction. It implies one's pre-existing opinion is 'wrong', which threatens participants' self-esteem, hence arousing negative emotions. Thus, those with this perception will try to reject it in order to defend their opinion. However, what if we present the counter-stereotypic information of a positive stereotype of an ingroup and negative stereotype of an outgroup? Are the perceptions still activated as a negative emotion? According to self-affirmation theory, individuals tend to build a positive image of themselves and the ingroup to boost their self-worth, confidence, and security. Research suggests that self-affirmation can improve personal identity and group-affirmation can strengthen social identity (Derks, Laar, \& Ellemers, 2009). The counter-stereotypic information here is equivalent to a sort of praise and honour, which supports individuals' collective self-esteem. Therefore, we wonder which is more important for the preservation of an individual's self-worth: to maintain his or her pre-existing stereotype, or to hear others' opinions of ingroup praise and outgroup dispraise and thereby change their pre-existing negative stereotype of the ingroup and positive stereotype of the outgroup. We conjecture that individuals might unconsciously change their pre-existing stereotype, which is associated with the motive of self-worth enhancement. 
Reading stereotype-confirming information can influence participants to perceive that their stereotypes are supported by others and their confidence might be strengthened and thus enhance the implicit outgroup favouritism, and therefore explicit counter-stereotypic cognition might fade away due to the inclination of selfworth and self-esteem protection. However, implicit ingroup derogation might not be enhanced after reading ingroup-derogating information, although on the other hand, it might, because the ingroup derogation is adverse to self-worth and self-esteem.

It was hypothesised that although general stereotypes were hardly changed when encountering extreme examples, stereotypes relevant to ingroup derogation and outgroup favouritism were likely to be changed easily towards the orientation of self-worth sustain. In Study 3, we investigated the effect of exposure of media information on participants' implicit and explicit group bias by presenting blogs that were either consistent or inconsistent with the implicit stereotype from the hypotheses of Study 2. The following hypotheses were tested:

Hypothesis 3a: Exposure to stereotype-confirming (ingroup derogation and outgroup favouritism) examples will enhance implicit outgroup favouritism but not ingroup derogation, and dissolve but not reverse the explicit ingroup favouritism and outgroup derogation.

Hypothesis 3b: Exposure to stereotype-disconfirming (ingroup favouritism and outgroup derogation) examples will dissolve both implicit ingroup derogation and outgroup favouritism, whereas it will not change the explicit ingroup favouritism and outgroup derogation.

\section{Materials and Methods}

The single factor of the between-subject design was exposure to stereotype-relevant illustrations, either implicit stereotype-confirming (ingroup derogation and outgroup favouritism), which was verified in Study 2, or implicit stereotype-disconfirming (ingroup favouritism and outgroup derogation), with no exposure for the control group. As dependent variables, the intensity of the implicit and explicit stereotype was measured by the SEB measure and self-reporting scale.

Participants. Chinese internet users $(N=113)$ were recruited as participants for free via the internet. After eliminating invalid questionnaires (i.e., those that were finished in less than 3 minutes, or were filled with irrelevant words, punctuations, or copies of the item sentences), 96 were available ( 53 men, 43 women).

Design and procedure. Under one condition of the experimental group, the blog contained five descriptions, two pictures and negative evaluations of Chinese uncivilised behaviours, and the same amount of illustrations plus positive evaluations of foreigners' civilised behaviours, which was consistent with the implicit stereotype found in Study 2 (ingroup derogation and outgroup favouritism). Un- der another condition, the blog contained five descriptions, two pictures and positive evaluations of Chinese civilised behaviours and the same amount of illustrations plus negative evaluations of foreigners' uncivilised behaviours, which was inconsistent with the implicit stereotype found in Study 2 (outgroup derogation and ingroup favouritism). The number of words for each (un)civilised behaviour and positive/negative evaluations was adjusted to avoid significant differences. There was no exposure to the blog for the control group. The materials for stereotype measurement were the same as those used in Study 2.

It is noteworthy that in order to simulate the real-life situation and guarantee a high external validity, Study 3 adopted examples selected from real news reports that had been analysed in Study 1 and were demonstrated to be extreme. An 11-point Likert scale of evaluation of civilisation degree of the actors in materials was used to validate the extremity of the experimental material, similar to a scale used by Kunda and Oleson (1997).

When participants opened the web page for the experiment, an introduction emphasising a non-normal answer was presented as in Study 2. Then participants were randomly assigned to an experimental condition to read a blog that was consistent or inconsistent with ingroup derogation and outgroup favouritism, or to a control group without a blog presentation.

The experimental groups would see a box saying 'Please read this famous blog', and then had at least 5 minutes to read the blog before proceeding to a questionnaire page, while the control group directly answered the questionnaire without this section. The presentation order was the SEB measure, self-report scale, and the demography questionnaire. The available SEB data was coded and scored with the same method used by the same coders in Study 2. After the scoring, 85 participants agreed to continue to rate the civilisation degree of the actors in experimental materials on an 11-point Likert scale.

\section{Results}

Data from 96 valid questionnaires (53 male, 43 are female) were analysed using SPSS16.0, and all statistical tests were conducted at the $p<.05$ significance level. As expected, the civilisation degree of actors in the experimental material was rated as extreme, which for all of them was much higher than the neutral line. Thus, the manipulation of the target extremity was effective.

Chinese intergroup bias in the implicit SEB measure. The ANOVA for the implicit stereotype revealed that exposure to stereotype-relevant examples had a significant effect on participants' implicit stereotypes of civilised behaviours of Westerners, $F(2,93)=3.202, p<.05, \eta^{2}=.06$, but not on implicit stereotypes of uncivilised behaviours of Chinese, $F(2,93)=1.021, p=.364$. According to the result of the multiple comparison tests, SEB1 (ingroup derogation), scores of participants in the stereotype-confirming condition (ingroup derogation and outgroup favouritism) 
were significantly higher than scores of participants in the other two conditions.

A sample $t$ test suggested that the SEB1 (ingroup derogation) and SEB2 (outgroup favouritism) under stereotype-confirming conditions (ingroup derogation and outgroup favouritism) were significantly higher than zero, $t_{1}(29)=3.807, p_{1}<.001, t_{2}(29)=2.829$, $p_{2}<.008$. In a control condition, the difference between SEB1 (ingroup derogation) and zero was marginally significant, $t(37)=1.957, p<.06$, and SEB2 (outgroup favouritism) was significantly higher than zero, $t(37)=$ $4.744, p<.001$. The SEB1 (ingroup derogation) and SEB2 (outgroup favouritism) of participants who received exposure to stereotype-disconfirming (ingroup favouritism and outgroup derogation) examples had no significant difference with zero, $t_{1}(27)=1.355, p_{1}=.187, t_{2}(29)=$ $1.585, p_{2}=.125$.

As predicted, the results of the control condition in this study indicated that implicit outgroup favouritism and ingroup derogation pre-existed in Chinese participants. The result of the ANOVA suggested that stereotype-confirming (ingroup derogation and outgroup favouritism) information enhanced implicit outgroup favouritism but not ingroup derogation, which supported Hypothesis 2a. And the result of the $t$ test showed that exposure to stereotypedisconfirming (ingroup favouritism and outgroup derogation) examples dissolved both the implicit outgroup favouritism and the ingroup derogation to be not significantly differ from zero, which supported Hypothesis $2 \mathrm{~b}$.

Chinese intergroup bias in the explicit self-report scale. The ANOVA showed the main effect of experimental manipulation with gender as a covariate on explicit group bias was significant, $F(2,93)=3.477, p<.04, \eta^{2}=.07$. A sample $t$ test suggested that explicit stereotype scores under the stereotype-disconfirming (ingroup favouritism and outgroup derogation) condition and control condition were significantly less than the theoretical mean of 3.5 (Chinese were more civilised than Westerners), $t(27)=$ $-2.324, p<.03$, for the stereotype-disconfirming condition, and $t(37)=-3.248, p<.002$, for the control condition. Whereas participants who received exposure to the stereotype-confirming condition (ingroup derogation and outgroup favouritism) had no significant difference at 3.5 (Chinese were as civilised as Westerners), $t(29)=$ $0.208, p_{2}=.837$, with the mean even higher at 3.547.

\section{Discussion}

Stereotype and group bias change of stereotypeconfirming condition. As predicted, exposure to the blog that confirmed the pre-existing implicit group bias (ingroup derogation and outgroup favouritism) enhanced implicit outgroup favouritism but not ingroup derogation, and dissolved but did not reverse the explicit ingroup favouritism and outgroup derogation.

Our results were partly consistent with of Stangor, Sechrist, and Jost's (2001) study, which found that stereo- types can be changed by consensus. In the present study, reading a blog article was equivalent to processing information from public opinions. Reading stereotypeconfirming information (ingroup derogation and outgroup favouritism) perhaps made participants perceive that their stereotypes were supported by the public voice, and thus enhanced the implicit outgroup favouritism and made explicit ingroup favouritism and outgroup derogation fade away. However, that implicit ingroup derogation was not enhanced might be due to self-worth and selfesteem. Exposure to stereotype-confirming information (ingroup derogation and outgroup favouritism) might arouse negative emotions. Here, participants perhaps did not have the sense of high self-worth when their preexisting implicit group bias was supported, and hence the negative stereotype of the ingroup would not be increased. The ingroup favouritism and outgroup derogation at an explicit level was vanished but not reversed, an explanation of which might be the inclination of self-worth and self-esteem protection as well.

Another possible explanation for the media stereotype-confirming (ingroup derogation and outgroup favouritism) information's influence is based on the media dependency theory (Ball-Rokeach \& DeFleur, 1976) that the impact of media information increases when other sources of knowledge (e.g., contact with outgroup members) are lacking. Since most ordinary Chinese people have few opportunities to have direct contact with Westerners, the mass media is their main source of knowledge about Westerners. So, compared to the stereotype of the ingroup, the mass media might have more impact on Chinese participants' stereotype of the outgroup.

Stereotype and group bias change of stereotypedisconfirming condition. Exposure to the blog information (ingroup favouritism and outgroup derogation) that disconfirmed the pre-existing implicit group bias dissolved both implicit ingroup derogation and outgroup favouritism, whereas it did not change the explicit ingroup favouritism and outgroup derogation.

Many researchers have demonstrated that stereotypedisconfirming information occurs only when people believe it (e.g., Kunda \& Oleson, 1997). And people are more likely to believe in the information propitious to self-worth, due to the a self-serving motivation of and self-enhancement. In the current study, the 'desired belief' was opposite of the pre-stereotype. It was exactly what the stereotype-disconfirming (ingroup favouritism and outgroup derogation) information exhibited. So, participants might tend to believe in the stereotypedisconfirming information that served self-enhancement and ingroup value.

Furthermore, many studies have demonstrated that stereotype-disconfirming information could arouse negative emotions, thus making people maintain pre-existing stereotypes. Most of these pre-stereotypes are either negative outward or positive inward, which was supportive 
of self-worth (e.g., Stangor, Sullivan, \& Ford, 1991). In our study, presenting information that disconfirmed preexisting ingroup derogation and outgroup favouritism was equivalent to enhancing the ingroup and meanwhile debasing the outgroup, which was supportive to self-worth and consistent with the direction of social identification. Therefore, although information was inconsistent with the pre-existing group bias and even extreme, it was consistent with participants' expectations of self-enhancement, so it might aroused a positive emotion, thereby facilitated the group bias change toward the direction of self-worth and ingroup-value enhancement.

\section{General Discussion}

Both the media analysis and implicit SEB measure provide converging evidence that Chinese people hold the implicit stereotype that Westerners are more civilised than Chinese, whereas when explicitly measured, Chinese participants show a reversed intergroup perception that Chinese are more civilised than Westerners. These results are consistent with the previous findings about a disadvantaged group's intergroup bias on other developed dimensions. This consistency demonstrates that 'civilised behaviour' is a valid indigenous intergroup comparison dimension in Chinese society.

\section{Theoretical Contributions}

The current research provides an optional theoretical interpretation for why Chinese people exhibit intergroup bias. For Chinese intergroup biases on other dimensions, previous literature have provided some explanations, which yet have limitations (e.g., Ma-Kellams et al., 2011). In the present article, we argued that the possible explanation for our results is based on the representation of Chinese modern history and Confucian thought.

As the underlying structure for the formation of attitudes and opinions, social representations of history have a significant effect on perceptions and attitudes by privileging certain historically warranted social categories (Liu \& Atsumi, 2008). Chinese people's intergroup perceptions might be influenced by the representation of long-term economic poverty. In China, Confucianism is unquestionably the most influential thought, which forms the foundation of Chinese cultural values and still provides the basis for Chinese social norms. Civilised behaviour is the embodiment of propriety, which in Confucian thought is used to differentiate people with different social and economic status. And, considering the fact that in the representation of modern history, Chinese people perceive themselves as relatively disadvantaged compared to a Western outgroup; they tend to regard the ingroup as uncivilised and the Western outgroup as civilised.

The results of the implicit and explicit measures in the studies help interpret why the application of SIT failed to be extended to the implicit results. The results of the explicit measure indicated that Chinese people strive to maintain a positive image of their group. Chinese people could not accept the perception of being less civilised than Westerner at their conscious awareness. When evaluated at an explicit level, the normative pressure to avoid being seen as prejudiced or discriminatory were diffused. Meanwhile, participants wanted to meet the need of satisfying ego- and group-justifying motives. Nevertheless, when measured at an implicit level, such pressure no longer existed and thus people tend to reveal their 'true' attitude.

Our research also contributes to the methodology of content analysis by extending the SEB to nonexperimental research. Previously, the SEB was limited to experimental studies (e.g., Logel et al., 2009). In the current research, the SEB was used to quantitatively analyse the frequency of (internal and external) attributions for different behaviours. Since the media content analysis results in Study 1 have been demonstrated to be consistent with the implicit SEB results, this method is scientifically validated and can be used in future studies that apply media or social representation analysis.

\section{Practical Implications}

In addition to the theoretical contribution, our research also provides some suggestions for practical implications and social change that may benefit society and individuals. Biased ingroup derogation and outgroup favouritism of Chinese people might trigger many social psychological phenomena, such as lower level of identification with Chinese, less self-esteem in the presence of Westerners, and a perceived threat of being assimilated by Western culture. We propose two ways to eliminate these prejudices and improve Chinese civilised behaviour.

First, proper guidance from mass media could contribute to changing unfair stereotypes. The results of Study 3 showed that exposure to stereotype-disconfirming media information dissolved both implicit outgroup favouritism and ingroup derogation, and exposure to stereotype-confirming media information directed the stereotype change towards the derogating ingroup and favoured outgroup. These findings suggest that since few Chinese have direct contact with Westerners, the impression of Chinese people that Westerners are more civilised is heavily influenced by the mass media and not by systematic knowledge or reality. Thus, the mass media information plays an important role in stereotype change in China. However, the results of Study 1 indicate that journalists hold a negative stereotype of civilised behaviours of Chinese people. Chinese journalists, as the significant component of mass media, could be guided to to deliver information about civilised behaviours more fairly. Changing negative stereotypes about Chinese behaviours and paying more attention to the objective situation would help Chinese people adopt an appropriate perspective. Meanwhile, the State Administration of Radio Film and Television of China could improve censorship by better 
directing the mass media to reduce untruthful and subjective reports as much as possible.

Second, more emphasis should be given to the propaganda of Chinese traditional virtues and cultures, such as Confucian thoughts, when (un)civilised behaviours are reported. The process of stereotype change connects tightly with the emotion of viewers. Normally, perceivers will try to reject information that contradicts pre-existing stereotypes in order to defend the stereotype to be changed. The results of Study 3 demonstrate that Chinese people are willing to accept mass media information of ingroup praise and outgroup dispraise and thereby change pre-existing stereotypes. In this case, the counterstereotypic information is a kind of praise and honour. Individuals unconsciously change their pre-existing stereotypes out of collective self-esteem and self-worth enhancement. There are a many good traditional virtues and cultures in China that Chinese people are proud of, such as Confucian philosophy. These national virtues and cultures could promote positive emotions in Chinese people. Similarly, Chinese people might feel ashamed when committing some uncivilised behaviours that are contrary to their national virtues and culture. Thus, adding more content about Chinese traditional virtues and culture when reporting (un)civilised behaviours would help improve civilised behaviour and inhibit uncivilised behaviour of Chinese people.

\section{Limitation and Future Research}

Limitations in the present research suggest some meaningful directions for future study. First, in the current study, civilised behaviour only served as a comparison dimension for two large groups (Chinese and Western). Considering that propriety is assumed to be associated with economic and social status, civilised behaviour as the behavioural embodiment of propriety can also be extended to other intergroup research related to economic, educational, and social status or power. Second, social representations of history proposed as the theoretical interpretation for Chinese dual intergroup bias on civilised behaviour need further direct empirical support. Future studies would benefit by studying how the priming of Confucian ideologies influence the belief that the higher the social and economic status of the actors, the more civilised they will be.

\section{Declaration of Interest}

The authors declare that there is no conflict of interest.

\section{Acknowledgments}

Special thanks to Professor James Liu from Victoria University of Wellington, Professor Craig Hart and Ms Joyce Adams from Brigham Young University, and Professor Ivana Markova from University of Stirling for their constructive and professional suggestions in revising this paper. This work was supported by the Major Project of
National Social Science Foundation (grant number 12\& ZD228 and 13\& ZD073).

\section{References}

Ball-Rokeach, S.J., \& DeFleur, M.L. (1976). A dependency model of mass media effects. Communication Research, 3, 3-21.

Bu, G. (2007). Origins of civilization in China. Beijing, China: Science Press.

Chinese Foreign Ministry. (2007, August 27) Chinese people's uncivil behavior cause increasing reminder abroad [video file]. Retrieved from http://news.ifeng.com/mainland/ 200708/0827_17_203930.shtml

Chao, M.M., \& Kung, F.Y. (2014). Culture and everyday sense making. Asian Journal of Social Psychology, 17, 112-114.

Chao, M.M., \& Kung, F.Y. (2015). An essentialism perspective on intercultural processes. Asian Journal of Social Psychology, $18,91-100$.

Cuddy, A.J.C., Fiske, S.T., Kwan, V.S.Y., Demoulin, S., Leyens, J., Bond, M.H., ... Ziegler, R. (2009). Stereotype content model across cultures: Towards universal similarities and some differences. British Journal of Social Psychology, 48, 133.

Dixon, T.L. (2006). Psychological reactions to crime news portrayals of Black criminals: Understanding the moderating roles of prior news viewing and stereotype endorsement. Communication Monographs, 73, 162-187.

Derks, B., van Laar, C., \& Ellemers, N. (2009). Working for the self or working for the group: How self versus group affirmation affects collective behavior in low-status groups. Journal of Personality and Social Psychology, 96, 183-202.

Du, M.D. (2006). Xun Zi's ideas of the ranks of rituals and their class nature. Chinese Culture Research, 1, 56-65.

Evett, S.R., Devine, P.O., Hirt, E.R., \& Price, J. (1994). The role of the hypothesis and the evidence in the trait hypothesis testing process. Journal of Experimental Social Psychology, $30,456-481$.

Frewin, K., Pond, R., \& Tuffin, K. (2009). Sexual abuse, counselling and compensation: discourses in New Zealand newspapers. Feminism \& Psychology, 19, 29-47.

Gibson, R., \& Zillman, D. (1993). The impact of quotations in news reports on issue perception. Journalism Quareterly, 70, 793-800.

Gonsalkorale, K., von Hippel, W., Sherman, J.W., \& Klauer, K.C. (2009). Bias and regulation of bias in intergroup interactions: Implicit attitudes toward Muslims and interaction quality. Journal of Experimental Social Psychology, 45, 161-166.

Haslam, S.A., Oakes, P.J., McGarty, C., Turner, J.C., Reynolds, K.J., \& Eggins, R.A. (1996). Stereotyping and social influence: The mediation of stereotype applicability and sharedness by the views of ingroup and outgroup members. British Journal of Social Psychology, 35, 369-397.

Hastie, R. (1984). Causes and effects of causal attribution. Journal of Personality and Social Psychology, 46, 44-56.

Haye, A., González,, R., Ordóñez,, G., Bohner,, G., Siebler, F., \& Sirlopú, D. (2010). System-perpetuating asymmetries between explicit and implicit intergroup attitudes among in- 
digenous and non-indigenous Chileans. Asian Journal of Social Psychology, 13, 163-172.

Heider, F. (1958). The psychology of interpersonal relations. New York: John Wiley \& Sons.

Hewstone, M., \& Ward, C. (1985). Ethnocentrism and causal attribution in Southeast Asia. Journal of Personality and Social Psychology, 48, 614-623.

Jost, J.T., Pelham, B.W., \& Carvallo, M. (2002). Non-conscious forms of system justification: Cognitive, affective, and behavioral preferences for higher status groups. Journal of Experimental Social Psychology, 38, 586-602.

Kashima, Y. (2014). Meaning, grounding, and the construction of social reality. Asian Journal of Social Psychology, 17, 81-95.

Kulik, J.A. (1983). Confirmatory attribution and the perpetuation of social beliefs. Journal of Experimental Social Psychology, 44, 1171-1181.

Kunda, Z., \& Oleson, K.C. (1995). Maintaining stereotypes in the face of disconfirmation: Constructing grounds for subtyping deviates. Journal of Personality and Social Psychology, 68, 565579 .

Kunda, Z., \& Oleson, K.C. (1997). When exceptions prove the rule: How extremity of deviance determines deviants' impact on stereotypes. Journal of Personality and Social Psychology, 72, 965-979.

Lam, S.K., Ahearne, M. Hu, \& Y. Schillewaert, N. (2010). Resistance to brand switching when a radically new brand is introduced: A social identity theory perspective. Journal of Marketing, 74, 128-146.

Leach, C., Ellemers, N., \& Barreto, M. (2007). Group virtue: The importance of morality (vs. competence and sociability) in the positive evaluation of in-groups. Journal of Personality and Social Psychology, 93, 234-249.

Liu, J.H. (2014). Extending Kashima's social construction of reality through the common ground of institutional communication and actors in social roles. Asian Journal of Social Psychology, 17, 96-99.

Liu, Z.L. (2011, February 2). National image film is popular in America, travel agencies call for civil travels. Fanyu Daily. Retrieved from: http://pyrb.dayoo.com/html/ 2011-02/02/content_1260209.htm

Liu, J.H., \& Atsumi, T. (2008). Historical conflict and resolution between Japan and China: Developing and applying a narrative theory of history and identity. In T. Sugiman, K.J. Gergen, W. Wagner, Y. Yamada (Eds.), Meaning in action: Constructions, narratives, and representations (pp. 327-344). Tokyo: Springer-Verlag.

Liu, J.H., Li, M., \& Yue, X. (2010). Chinese social identity and intergroup relations: The influence of benevolent authority. In M.H. Bond (Ed.), Oxford handbook of Chinese psychology (2nd ed., pp. 579-597). Oxford: Oxford University Press.

Logel, C., Walton, G.M. Logel, Spencer, , S.J. Spencer, Iserman, E.C. Iserman, von Hippel, W., \& Bell, A.E. (2009). Interacting with sexist men triggers social identity threat among female engineers. Journal of Personality and Social Psychology, 96, 1089-1103.

Ma-Kellams, C., Spencer-Rodgers, J., \& Peng, K. (2011). I am against us? Unpacking cultural differences in ingroup fa- voritism via dialecticism. Personality and Social Psychology Bulletin, 37, 15-27.

Maner, J.K., Miller, S.L., Moss, J., Leo, J., \& Plant, E.A. (2012). Motivated social categorization: Fundamental motives enhance people's sensitivity to basic social categories. Journal of Personality and Social Psychology, 103, 7083.

Munro, G.D., \& Ditto, P.H. (1997). Biased assimilation, attitude polarization, and affect in reactions to stereotype-relevant scientific information. Personality and Social Psychology Bulletin, 23, 636-653.

Pál,, N. (2009). Between encouragement and control: Tourism, modernity and discipline in China. In T. Winter, P. Teo, \& T.C. Chang (Eds.), Asia on tour: exploring the rise of Asian tourism (pp. 158-159). New York: Routledge Press.

Stangor, C., Sechrist, G.B., \& Jost, J.T. (2001). Changing racial beliefs by providing consensus information. Personality and Social Psychology Bulletin, 27, 486-496.

Stangor, C., Sullivan, L.A., \& Ford, T.E. (1991). Affective and cognitive determinants of prejudice. Social Cognition, 9, 359381.

Tajfel, H., \& Turner, J.C. (1986). The social identity of intergroup behavior. In S. Worchel \& W.G. Austin (Eds.), Psychology of Intergroup Relations (pp. 7-24). Chicago, IL: Nelson-Hall Publishers.

Tomba, L. (2009). Of quality, harmony, and community: Civilization and the middle class in urban China. Positions, 17, 591-616.

Rudman, L.A., Greenwald, A.G., \& McGhee, D.E. (2001). Implicit self-concept and evaluative implicit gender stereotypes: Self and ingroup shares desirable traits. Personality and Social Psychology Bulletin, 9, 1164-1178.

Von Hippel, W., Sekaquaptewa, D., \& Vargas, P. (1997). The linguistic intergroup bias as an implicit indicator of prejudice. Journal of Experimental Social Psychology, 33, 490509.

Wan, X.J. (2009, January 5). What did Wall Street Bull reveal and who is the black sheep in China? China Value online forum. Retrieved from http://www.chinavalue.net/Group/Topic/19258/.

Wang, S.M. (2004). Elaboration of Confucius ethics thought. Jinan, China: Qilu Books.

Wang, F. (2007). A group of Chinese tourists actually ride the charging bull in Wall Street! Sina Blog. Retrieved November 15, 2007, from http://blog.sina.com.cn/wangfang.

Weber, R., \& Crocker, J. (1983). Cognitive processes in the revision of stereotypic beliefs. Journal of Personality and Social Psychology, 45, 961-977.

Wilder, D.A. (1993). The role of anxiety in facilitating stereotypic judgments of outgroup behavior. In D.M. Mackie, \& D.L. Hamilton (Eds.), Affect cognition and stereotyping: Interactive processes in group perception (pp. 87-109). Academic Press: San Diego.

Wilder, D.A., Simon, A.F., \& Faith, M. (1996). Enhancing the impact of counter stereotypic information: Dispositional attributions for deviance. Journal of Personality and Social Psychology, 71, 276-287. 